\title{
Métodos de análise em programas de segurança alimentar e nutricional: uma experiência no Brasil
}

\author{
Methods of analysis of food and nutritional security programs: \\ an experience in Brazil
}

Alice Teles de Carvalho ${ }^{1}$

Erika Rodrigues de Almeida ${ }^{1}$

Eduardo Augusto Fernandes Nilson ${ }^{2}$

Juliana Amorim Ubarana ${ }^{3}$

Irela Mazar Fernández ${ }^{4}$

Maarten Immink ${ }^{4}$

\footnotetext{
${ }^{1}$ Departamento de Nutrição Centro de Ciências da Saúde - Campus I, Universidade Federal da Paraíba. Campus Universitário. s/n. CCS. $2^{\circ}$ andar. Depto. Nutrição, Castelo Branco III. 58059-900 Joao Pessoa PB. alicetel@terra.com.br ${ }^{2}$ Coordenação Geral de Alimentação e Nutrição, Ministério da Saúde do Brasil.

${ }^{3}$ Organização

Pan-americana de Saúde. ${ }^{4}$ Organização das Nações Unidas para Agricultura e Alimentação.
}

\begin{abstract}
The development of methods that enable analysis of programs and public policies is a key point for their objectives to be attained. This study presents a Brazilian experience of analysis of two food and nutritional security programs in cities in Brazil's northeast region, namely the National Program for Vitamin A Supplementation, and monitoring health conditions of those included in the Family Allowance Program. The methodological stages included the definition of programs to be analyzed, data collection locations, the population participating in the research, collection procedures and data analysis. The methodology used made it possible to ascertain the perception of the different social actors involved in the operationalization of these programs, proving to be an important tool for the insertion of these actors, not only as objects of analysis, but also mainly as subjects of self-evaluation.
\end{abstract}

Key words Evaluation of research programs and tools, Nutrition programs and policies, Methods
Resumo O desenvolvimento de métodos que permitam analisar programas e políticas públicas é ponto chave para que os seus objetivos sejam alcançados. Este estudo apresenta uma experiência brasileira de análise de dois programas de segurança alimentar e nutricional, em municípios do nordeste do país: Programa Nacional de Suplementação de Vitamina A e acompanhamento das condicionalidades de saúde do Programa Bolsa Família. Adaptou-se metodologia proposta pela Organização das Nações Unidas para Agricultura e Alimentação. As etapas metodológicas compreenderam a definição dos programas a serem analisados, dos locais de coleta de dados, do público participante da pesquisa e dos procedimentos de coleta e análise dos dados. A metodologia utilizada permitiu apreender a percepção dos diferentes atores sociais envolvidos na operacionalização dos programas acerca destes, mostrando-se como uma importante ferramenta de inserção destes atores não apenas como objetos de análise, mas principalmente como sujeitos de autoavaliação.

Palavras-chave Avaliação de programas e instrumentos de pesquisa, Programas e políticas de nutrição e alimentação, Métodos 


\section{Introdução}

É notório que tanto a saúde como a nutrição dependem de uma gama de determinantes que abarcam condições socioeconômicas, culturais e ambientais. Assim, a má nutrição, em todas as suas formas, sobrecarrega não apenas os sistemas de saúde nacionais, mas todo o tecido cultural, social e econômico das nações, e é um dos impedimentos à concretização do potencial humano ${ }^{1}$.

A busca da equidade e o compromisso com uma atuação efetiva e eficiente sobre os determinantes responsáveis pela geração de padrões inadequados de alimentação e, consequentemente, de nutrição e saúde das pessoas, é tarefa a ser construída pelos governos e sociedades e por meio do entendimento entre países ${ }^{2}$.

Em 1992, a Conferência Internacional sobre Nutrição, realizada em Roma, trouxe à tona a necessidade da implementação de estratégias básicas de alimentação, nutrição, saúde e bem-estar das populações ${ }^{3}$.Em 1996, a Cúpula Mundial de Alimentação aprovou a Declaração de Roma sobre Segurança Alimentar e o Plano de Ação, sinalizando a importância de identificar as causas da fome e da má nutrição, assim como, de adotar medidas que ajudem a melhorar a situação ${ }^{4}$. Em 2002, a reunião da Cúpula Mundial de Alimentação reafirmou os compromissos para eliminar a fome e convocou a formação de uma aliança internacional, com o fim de acelerar os esforços para alcance desse objetivo ${ }^{5}$. Desde então, os países vêm elaborando planos nacionais de ação para a nutrição e a segurança alimentar, tendo como objetivo o enfrentamento de problemas relacionados a este tema.

Apesar de grande parte dos países implementar programas e ações de alimentação e nutrição, em geral ainda não são realizadas ações institucionalizadas de monitoramento e avaliação voltadas a aperfeiçoá-los. Diversos estudos têm, inclusive, apontado a necessidade do desenvolvimento de uma metodologia de análise que permita o aperfeiçoamento desses programas, com vistas à melhoria de seus resultados, sua rentabilidade, eficácia e sustentabilidade ${ }^{6,7}$.

No sentido de contribuir com o fortalecimento da capacidade analítica dos países, com relação aos programas de Alimentação e Nutrição comunitários, a Organização das Nações Unidas para Agricultura e Alimentação (FAO) iniciou um processo que culminou no desenvolvimento de um Instrumento de Análise de Programas de Nutrição.

Para entender melhor os fatores que fazem os programas de alimentação e nutrição comu- nitários serem efetivos, eficientes, equitativos e sustentáveis, ou seja, os fatores determinantes de seu êxito, a FAO realizou nove estudos de caso na América, África e Ásia. Esses estudos forneceram informações sobre lições aprendidas, aspectos positivos e limitações dos programas de nutrição, que foram analisadas e consolidadas em uma oficina realizada em Roma, em outubro de $2001^{8}$.

Em junho de 2002 foi realizada uma nova oficina, dessa vez na Cidade do Cabo, na África do Sul, com o propósito de promover o debate do conteúdo apresentado, preliminarmente, no texto do Instrumento de Análise dos Programas de Nutrição. Ao final do evento, foram incorporadas as modificações no texto do instrumento com base nas recomendações da oficina. Com as lições aprendidas dos referidos estudos, a FAO elaborou o guia metodológico "Melhora dos programas de Nutrição: Um instrumento de análise para a ação (IA)" "9.

Em 2007, a FAO criou um projeto para pôr em prática o uso do instrumento de análise de programas de nutrição em quatro de seus países membros (Argentina, Brasil, México e Panamá), visando à troca de experiências e conhecimentos entre os partícipes.

No Brasil, o projeto Análise e Fortalecimento de Programas de Alimentação e Nutrição Comunitária incluiu como objeto de estudo dois programas estratégicos à promoção da segurança alimentar e nutricional (SAN), o Programa Nacional de Suplementação de Vitamina A (PNVITA) e o acompanhamento das condicionalidades do setor saúde do Programa Bolsa Família (PBF). Estes programas compõem o rol de estratégias do governo federal brasileiro para a garantia da SAN da população. O Brasil tem se destacado por possuir uma rede de instituições governamentais e não governamentais voltadas à execução da política de alimentação e nutrição existente, na perspectiva do alcance da SAN e do respeito ao Direito Humano a Alimentação Adequada (DHAA). No entanto, ações de acompanhamento e avaliação destas estratégias ainda não estão incorporadas de forma institucionalizada à rede de atenção a saúde.

A participação do Brasil neste projeto teve como objetivo fortalecer os programas de SAN e manter no cenário nacional o debate acerca da necessidade de institucionalização das ações de monitoramento e avaliação.

Este trabalho traz a experiência de aplicação da metodologia IA-FAO de análise no Brasil, descrevendo o caminho metodológico percorrido para identificar a forma de operacionalização do 
PNVITA e do acompanhamento das condicionalidades do setor saúde do PBF, destacando as limitações e dificuldades, assim como as potencialidades, para traçar um plano de ação na perspectiva de aperfeiçoamento.

\section{Método}

A experiência aqui relatada tem como base a metodologia IA proposta pela $\mathrm{FAO}$, cujo objetivo é aperfeiçoar a análise dos programas de alimentação e nutrição comunitários, sua eficiência e seu impacto na população participante, bem como fortalecer a capacidade analítica dos técnicos dos programas.

O IA baseia-se na metodologia DAFO, pois recomenda que o processo de avaliação aponte as Debilidades, Ameaças/limitações, Fortalezas e Oportunidades em cada item analisado9. Está dividido em quatro seções que abordam o desenho do programa, o macroentorno, o microentorno e a sustentabilidade (Figura 1).

A primeira aproximação dos países participantes com a metodologia proposta pela FAO se deu em uma oficina de trabalho realizada na cidade de Brasília, Brasil, em que participaram os coordenadores e osconsultores dos projetos em cada país.

No Brasil, a execução da pesquisa ficou a cargo da Coordenação Geral de Alimentação e Nutrição do Ministério da Saúde (CGAN/MS), em parceria com o Departamento de Nutrição da Universidade Federal da Paraíba (UFPB).
Após a oficina de aproximação dos países com a metodologia IA-FAO de análise, a equipe de condução do projeto definiu os programas a serem avaliados no Brasil, os locais onde a coleta de dados seria realizada, o público participante da pesquisa e a metodologia a ser utilizada para a coleta e análise dos dados.

\section{Definição dos programas a serem avaliados}

Os programas brasileiros selecionados para avaliação foram o PBF, especificamente o acompanhamento das condicionalidades de saúde, e o PNVITA. Para a escolha dos programas, considerou-se a sua importância como estratégia de SAN e o seu nível de institucionalização (programas normatizados pelo Governo).

O PBF corresponde a um programa de transferência direta de renda com condicionalidades, que beneficia famílias em situação de pobreza e extrema pobreza. Configura-se como o principal instrumento da Estratégia Fome Zero, a qual está inserida no contexto da promoção da SAN, buscando a inclusão social e a conquista da cidadania da população mais vulnerável à fome $\mathrm{e}^{10,11}$. As condicionalidades do PBF no setor saúde englobam o acompanhamento do crescimento e desenvolvimento infantil, a vacinação de crianças menores de sete anos de idade e a atenção pré-natal e no pós-parto ${ }^{12}$.

Já o PNVITA, que é reconhecido na Política Nacional de Alimentação e Nutrição (PNAN) como uma das estratégias de SAN, está relacionado ao

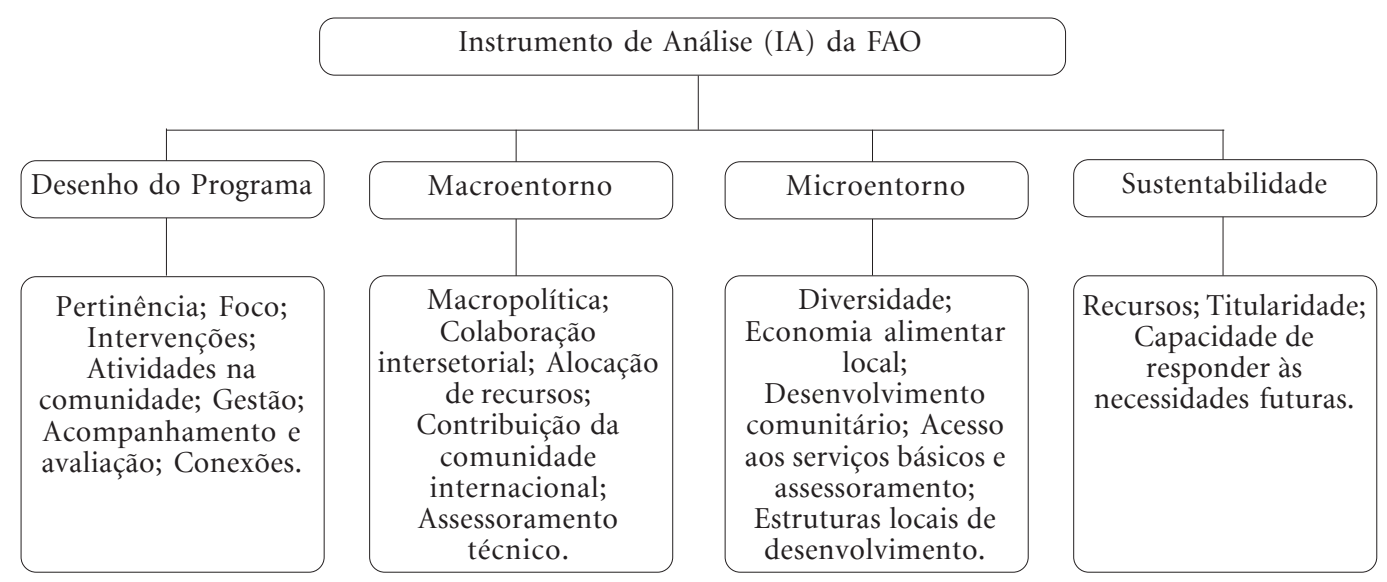

Figura 1. Seções do Instrumento de Análise da FAO, com seus respectivos itens de análise - dados originados do IA-FAO'. Brasil, 2008. 
controle da deficiência de vitamina A, identificada como um problema de saúde pública em mais de 120 países $^{13}$. O controle desta deficiência é realizado por meio da suplementação com cápsulas de megadose de vitamina $A$ às crianças de 06 a 59 meses e puérperas no pós-parto imediato. O Brasil é considerado área de carência subclínica grave, apresentando uma situação endêmica em grandes espaços das regiões Norte, Nordeste e Sudeste ${ }^{14-18}$. As áreas consideradas de risco para a deficiência de vitamina A e que são contempladas com o PNVITA são a região Nordeste, o Estado de Minas Gerais (região norte, Vale do Jequitinhonha e Vale do Mucurici) e o Vale do Ribeira, em São Paulo ${ }^{19}$.

\section{A escolha dos locais \\ para análise dos programas}

Decidiu-se desenvolver a pesquisa avaliativa em municípios da região Nordeste do País, em virtude desta apresentar uma menor proporção de indivíduos em situação de $\mathrm{SAN}^{20}$. Além disso, um dos programas selecionados para análise $-\mathrm{o}$ PNVITA - tem o seu funcionamento concentrado nesta região. Assim, as Coordenações Estaduais de Alimentação e Nutrição foram contatadas pela CGAN para decisão conjunta dos municípios para a realização da pesquisa.

Para o PBF, considerou-se importante a seleção de dois municípios situados em estados diferentes. O primeiro município deveria estar localizado em Alagoas, onde os indicadores de saúde e de educação demonstram profunda desigualdade; e o segundo município deveria estar no Rio Grande do Norte, onde há a maior cobertura de acompanhamento do PBF pelo setor saúde. Para esses dois municípios foram considerados os critérios de proximidade com a capital (por facilidade logística), de adesão dos secretários de saúde à proposta de avaliação do PBF e a existência de unidades de saúde dentro da proposta da Estratégia Saúde da Família (ESF).

Dessa forma, foram selecionados dois municípios: Tibau do Sul, no estado do Rio Grande do Norte, e Barra de São Miguel, no estado de Alagoas - para análise do acompanhamento das condicionalidades do setor saúde no PBF.

Para análise do PNVITA, o município de Cabedelo, no Estado da Paraíba, foi selecionado por possuir cobertura do programa passível de melhora e ser campo de estágio de disciplina coordenada por uma das pesquisadoras da equipe de condução do projeto, facilitando assim o acesso às informações, à identificação e ao treinamento da equipe de trabalho de campo.

\section{A definição do público participante da pesquisa, das técnicas \\ e dos instrumentos de coleta de dados}

Por tratar-se de programas de alimentação e nutrição comunitários, desenvolvidos especialmente no nível de Atenção Primária à Saúde ESF -, decidiu-se realizar uma pesquisa avaliativa participativa, utilizando o desenho de um estudo epidemiológico transversal, de base populacional, com abordagem aos diferentes atores sociais envolvidos na operacionalização dos programas: responsáveis por crianças participantes dos programas, profissionais de saúde e gestores.

A escolha da metodologia de pesquisa avaliativa participativa baseou-se no entendimento de que a execução adequada das atividades inerentes aos programas depende substancialmente da mobilização da população participante. Além disso, esta metodologia permite a apreensão de como as políticas e programas são percebidos pelo público-alvo, contribuindo para a promoção da integração objetiva e subjetiva, com a inserção dos atores sociais do programa não só como objetos de análise, mas, sobretudo, como sujeitos de autoavaliação ${ }^{21}$.

Os Quadros 1 e 2 apresentam, respectivamente, informações das etapas de coleta de dados para análise dos programas Bolsa Família e PNVITA. Nestes estão descritos o público participante da pesquisa, as técnicas de coleta de dados escolhidas para abordar cada grupo, os instrumentos elaborados e os eixos de abordagem de cada instrumento.

Foram realizadas entrevistas estruturadas e semiestruturadas, encontros de grupos focais, análise documental, observação in loco e avaliação do estado nutricional de crianças participantes dos programas.

As entrevistas foram utilizadas por permitirem abordagem de pessoas dos mais diversos graus de escolaridade, já que não exigem leitura nem escrita do entrevistado, pois as perguntas e as respostas são realizadas oralmente ${ }^{22}$. Optouse pelas entrevistas estruturadas em virtude de possibilitarem que os responsáveis por crianças menores de cinco anos fossem investigados simultaneamente nos três municípios cenário de estudo, abrangendo uma área geográfica mais ampla. Já as entrevistas semiestruturadas realizadas com os profissionais e gestores envolvidos com os programas foram utilizadas por viabilizarem maior aprofundamento de algumas questões, como os processos de operacionalização e gestão das ações realizadas. 
Quadro 1. Instrumentos elaborados para análise do acompanhamento das condicionalidades de saúde do Programa Bolsa Família (PBF), de acordo com o público participante, técnica de coleta de dados, instrumento elaborado e eixos de abordagem. Tibau do Sul (RN) e Barra de São Miguel (AL), Brasil, 2008.

\begin{tabular}{|c|c|c|c|}
\hline $\begin{array}{c}\text { Público } \\
\text { participante }(\mathbf{n})\end{array}$ & $\begin{array}{l}\text { Técnica de } \\
\text { coleta de dados } \\
\text { utilizada }\end{array}$ & $\begin{array}{l}\text { Instrumento } \\
\text { elaborado }\end{array}$ & Eixos de abordagem \\
\hline $\begin{array}{l}\text { Crianças menores } \\
\text { de cinco anos } \\
(\mathrm{n}=727)\end{array}$ & $\begin{array}{l}\text { Tomada de } \\
\text { medidas } \\
\text { antropométricas }\end{array}$ & $\begin{array}{l}\text { Formulário para } \\
\text { registro das } \\
\text { medidas de peso } \\
\text { e altura }\end{array}$ & Avaliação nutricional das crianças. \\
\hline $\begin{array}{l}\text { Responsáveis por } \\
\text { crianças menores } \\
\text { de } 5 \text { anos } \\
(\mathrm{n}=745)\end{array}$ & $\begin{array}{l}\text { Entrevista } \\
\text { estruturada }\end{array}$ & $\begin{array}{l}\text { Roteiro de } \\
\text { entrevista } \\
\text { estruturada }\end{array}$ & $\begin{array}{l}\text { - Acesso e utilização dos serviços de saúde; } \\
\text { - Perfil da família (nº moradores e composição); } \\
\text { - Vínculo com o PBF; } \\
\text { - Contribuições (impacto) do PBF na vida da família; } \\
\text { - Percepção sobre saúde e sobre o PBF; } \\
\text { - Conhecimento sobre o PBF (funcionamento e normas); } \\
\text { - Cobertura vacinal e avaliação nutricional das crianças. }\end{array}$ \\
\hline $\begin{array}{l}\text { Responsáveis por } \\
\text { crianças } \\
\text { participantes do } \\
\text { PBF } \\
(\mathrm{n}=29)\end{array}$ & $\begin{array}{l}\text { Encontro de } \\
\text { grupo focal }\end{array}$ & $\begin{array}{l}\text { Roteiro de grupo } \\
\text { focal }\end{array}$ & $\begin{array}{l}\text { - Vínculo com o PBF; } \\
\text { - Acesso ao PBF e aos serviços de saúde; } \\
\text { - Percepção sobre o PBF e sobre os serviços de saúde; } \\
\text { - Saúde como direito; } \\
\text { - Conhecimento sobre o PBF (funcionamento e normas); } \\
\text { - Contribuições (impacto) do PBF na vida da família; } \\
\text { - Sugestões para melhoria do PBF. }\end{array}$ \\
\hline $\begin{array}{l}\text { Profissionais de } \\
\text { saúde - médicos } \\
\text { (as), enfermeiros } \\
\text { (as) e técnicos (as) } \\
\text { de Enfermagem } \\
\quad(\mathrm{n}=21)\end{array}$ & $\begin{array}{c}\text { Entrevista } \\
\text { semiestruturada }\end{array}$ & $\begin{array}{c}\text { Roteiro de } \\
\text { entrevista } \\
\text { semiestruturada }\end{array}$ & $\begin{array}{l}\text { - Vínculo com a ESF e com o PBF; } \\
\text { - Capacitação sobre ESF e PBF; } \\
\text { - Conhecimento sobre o PBF; } \\
\text { - Percepção sobre o PBF e sobre os serviços de saúde; } \\
\text { - Papel no PBF; } \\
\text { - Sugestões para melhoria do PBF; } \\
\text { - Equidade em saúde. }\end{array}$ \\
\hline $\begin{array}{l}\text { Profissionais de } \\
\text { saúde - Agentes } \\
\text { comunitários de } \\
\text { saúde } \\
\quad(\mathrm{n}=27)\end{array}$ & $\begin{array}{l}\text { Encontro de } \\
\text { grupo focal }\end{array}$ & $\begin{array}{l}\text { Roteiro de grupo } \\
\text { focal }\end{array}$ & $\begin{array}{l}\text { - Capacitação e conhecimento sobre o PBF; } \\
\text { - Percepção sobre o PBF, pobreza e serviços de saúde; } \\
\text { - Vínculo com o PBF; } \\
\text { - Sugestões para melhoria do PBF. }\end{array}$ \\
\hline $\begin{array}{l}\text { Gestores (setor } \\
\text { saúde): } \\
\text { - Secretário de } \\
\text { saúde } \\
\text { - Coordenador da } \\
\text { Atenção Básica } \\
\text { - Coordenador do } \\
\text { PBF } \\
\text { - saúde } \\
\text { - Digitador } \\
\text {-máster do PBF } \\
\text { (n = 05) }\end{array}$ & $\begin{array}{c}\text { Entrevista } \\
\text { semiestruturada }\end{array}$ & $\begin{array}{l}\text { Roteiro de } \\
\text { entrevista } \\
\text { semiestruturada }\end{array}$ & $\begin{array}{l}\text { - Capacitação, conhecimento e percepção sobre o PBF; } \\
\text { - Operacionalização e gestão do PBF; } \\
\text { - Intersetorialidade; } \\
\text { - Recursos para o PBF; } \\
\text { - Controle social e participação popular; } \\
\text { - Política de avaliação e monitoramento; } \\
\text { - Vínculo com o PBF. } \\
\text { - Acesso ao PBF, aos serviços de saúde e à informação; } \\
\text { - Desempenho do PBF, dos serviços de saúde e destes frente ao PBF; } \\
\text { - Impacto do PBF nos serviços de saúde, na vida das famílias e na } \\
\text { relação destas com os serviços de saúde; } \\
\text { - Sugestões para melhoria do PBF e dos serviços de saúde; }\end{array}$ \\
\hline $\begin{array}{l}\text { Gestores (setor } \\
\text { ação social): } \\
\text { - Secretário de } \\
\text { ação social } \\
\text { - Coordenador do } \\
\text { PBF - ação social } \\
\quad(\mathrm{n}=04)\end{array}$ & $\begin{array}{c}\text { Entrevista } \\
\text { semiestruturada }\end{array}$ & $\begin{array}{l}\text { Roteiro de } \\
\text { entrevista } \\
\text { semiestruturada }\end{array}$ & $\begin{array}{l}\text { - Operacionalização, gestão e impacto do PBF; } \\
\text { - Conhecimento e capacitação sobre o PBF; } \\
\text { - Percepção sobre o PBF e sobre pobreza; } \\
\text { - Vínculo com o PBF; } \\
\text { - Perfil das famílias cadastradas no PBF; } \\
\text { - Recursos para o PBF; } \\
\text { - Intersetorialidade; } \\
\text { - Controle social e participação popular; } \\
\text { - Sugestões para melhoria do PBF; } \\
\text { - Desempenho do PBF e da ação social e saúde frente ao PBF; } \\
\text { - Direito à saúde; } \\
\text { - Acesso à informação. }\end{array}$ \\
\hline
\end{tabular}


Quadro 2. Instrumentos elaborados para análise do Programa Nacional de Suplementação de Vitamina A, de acordo com o público participante, técnica de coleta de dados, instrumento elaborado e eixos de abordagem. Cabedelo, Brasil, 2008.

\begin{tabular}{|c|c|c|c|}
\hline $\begin{array}{c}\text { Público } \\
\text { participante (n) }\end{array}$ & $\begin{array}{c}\text { Técnica de } \\
\text { coleta de dados } \\
\text { utilizada }\end{array}$ & $\begin{array}{l}\text { Instrumento } \\
\text { elaborado }\end{array}$ & Eixos de abordagem \\
\hline $\begin{array}{l}\text { Crianças menores } \\
\text { de cinco anos } \\
(\mathrm{n}=750)\end{array}$ & $\begin{array}{c}\text { Tomada de } \\
\text { medidas } \\
\text { antropométricas }\end{array}$ & $\begin{array}{l}\text { Formulário para } \\
\text { registro das } \\
\text { medidas de peso } \\
\quad \text { e altura }\end{array}$ & Avaliação nutricional das crianças. \\
\hline $\begin{array}{l}\text { Responsáveis por } \\
\text { crianças menores } \\
\text { de } 5 \text { anos } \\
(\mathrm{n}=780)\end{array}$ & $\begin{array}{l}\text { Entrevista } \\
\text { estruturada }\end{array}$ & $\begin{array}{l}\text { Roteiro de } \\
\text { entrevista } \\
\text { estruturada }\end{array}$ & $\begin{array}{l}\text { - Acesso e utilização dos serviços de saúde; } \\
\text { - Desempenho do PNVITA; } \\
\text { - Acesso ao PNVITA; } \\
\text { - Educação alimentar e nutricional; } \\
\text { - Percepção sobre o PNVITA, sobre a vitamina A e sobre saúde; } \\
\text { - Conhecimento sobre o PNVITA (funcionamento e normas) e } \\
\text { sobre a vitamina A; } \\
\text { - Cobertura de vitamina A. }\end{array}$ \\
\hline $\begin{array}{l}\text { Responsáveis por } \\
\text { crianças menores } \\
\text { de } 5 \text { anos }(n=15)\end{array}$ & $\begin{array}{l}\text { Encontro de } \\
\text { grupo focal }\end{array}$ & $\begin{array}{l}\text { Roteiro de grupo } \\
\text { focal }\end{array}$ & $\begin{array}{l}\text { - Acesso e utilização dos serviços de saúde; } \\
\text { - Acesso às ações de nutrição na Atenção Primária, a suplementos } \\
\text { vitamínicos e ao PNVITA; } \\
\text { - Educação alimentar e nutricional; } \\
\text { - Aleitamento materno; } \\
\text { - Percepção sobre o PNVITA; } \\
\text { - Consumo alimentar e acesso à alimentação; } \\
\text { - Conhecimento sobre o PNVITA (funcionamento e normas) e } \\
\text { sobre a vitamina A; } \\
\text { - Apoio assistencial; } \\
\text { - Participação popular e controle social; } \\
\text { - Saúde como direito; } \\
\text { - Sugestões para melhoria do PNVITA e do acesso à alimentação; } \\
\text { - Desempenho dos serviços de saúde. }\end{array}$ \\
\hline $\begin{array}{c}\text { Profissionais de } \\
\text { saúde - médicos } \\
\text { (as), enfermeiros } \\
\text { (as) e técnicos (as) } \\
\text { de Enfermagem } \\
(\mathrm{n}=22)\end{array}$ & $\begin{array}{c}\text { Entrevista } \\
\text { semiestruturada }\end{array}$ & $\begin{array}{c}\text { Roteiro de } \\
\text { entrevista } \\
\text { semiestruturada }\end{array}$ & $\begin{array}{l}\text { - Vínculo com a ESF e com o PNVITA (papel no programa); } \\
\text { - Conhecimento sobre as ações de nutrição na Atenção Primária, } \\
\text { sobre o PNVITA e sobre a vitamina A; } \\
\text { - Capacitação sobre o PNVITA e ESF; } \\
\text { - Percepção sobre o PNVITA e sobre os serviços de saúde; } \\
\text { - Acesso ao PNVITA; } \\
\text { - Operacionalização e desempenho do PNVITA; } \\
\text { - Saúde como direito; } \\
\text { - Sugestões para melhoria do PNVITA. }\end{array}$ \\
\hline $\begin{array}{l}\text { Profissionais de } \\
\text { saúde - Agentes } \\
\text { comunitários de } \\
\text { saúde }(\mathrm{n}=15)\end{array}$ & $\begin{array}{l}\text { Encontro de } \\
\text { grupo focal }\end{array}$ & $\begin{array}{l}\text { Roteiro de grupo } \\
\text { focal }\end{array}$ & $\begin{array}{l}\text { - Capacitação sobre o PNVITA; } \\
\text { - Acesso à alimentação; } \\
\text { - Desempenho do PNVITA; } \\
\text { - Vínculo (papel) com o PNVITA; } \\
\text { - Conhecimento sobre as ações de nutrição na Atenção Primária, } \\
\text { sobre o PNVITA e sobre a vitamina A; } \\
\text { - Sugestões para melhoria do PNVITA; } \\
\text { - Participação popular e controle social. }\end{array}$ \\
\hline $\begin{array}{l}\text { Profissionais de } \\
\text { saúde - } \\
\text { nutricionistas } \\
\text { inseridos nas } \\
\text { equipes de Saúde } \\
\text { da Família } \\
(\mathrm{n}=09)\end{array}$ & $\begin{array}{l}\text { Entrevista } \\
\text { estruturada }\end{array}$ & $\begin{array}{l}\text { Roteiro de } \\
\text { entrevista } \\
\text { estruturada }\end{array}$ & $\begin{array}{l}\text { - Operacionalização do PNVITA; } \\
\text { - Capacitação sobre o PNVITA; } \\
\text { - Conhecimento sobre as ações de nutrição na Atenção Primária, } \\
\text { sobre o PNVITA e sobre a vitamina A. }\end{array}$ \\
\hline
\end{tabular}

continu

Com relação aos encontros de grupos focais, optou-se pela utilização desta técnica com o intuito de estimular os participantes a discutirem sobre algumas questões abordadas nas entrevistas individuais, aprofundando os temas trabalhados e complementando os resultados já obti- 
Quadro 2. continuação

\begin{tabular}{|c|c|c|c|}
\hline $\begin{array}{l}\text { Público } \\
\text { participante (n) }\end{array}$ & $\begin{array}{c}\text { Técnica de } \\
\text { coleta de dados } \\
\text { utilizada }\end{array}$ & $\begin{array}{l}\text { Instrumento } \\
\text { elaborado }\end{array}$ & Eixos de abordagem \\
\hline
\end{tabular}

dos. Os grupos foram realizados segundo recomendação de Minayo $^{23}$, e gravados por meio de gravador digital, sempre com a presença de um moderador e um relator.

A tomada das medidas antropométricas foi realizada com o objetivo de identificar a situação nutricional das crianças participantes dos programas. Foi realizada a aferição do peso, com uso de balança digital com capacidade de até 150 $\mathrm{kg}$, e da estatura, utilizando-se dois modelos de antropômetro, sendo um para medir as crianças em pé e outro para a medição do comprimento de crianças de colo. Tanto para o peso quanto para a estatura foram realizadas duas medidas para cada criança, no sentido de minimizar os erros de aferição.

Os instrumentos de coleta de dados foram elaborados com base na literatura científica da área, incluindo portarias e decretos que regem ambos os programas, na metodologia IA-FAO, e nas discussões com as equipes da CGAN, do Centro Colaborador em Alimentação e Nutrição da Universidade Federal de Pernambuco e da Ação Brasileira pela Nutrição e Direitos Humanos.

A primeira versão dos instrumentos foi submetida a teste piloto realizado em Unidades de Saúde da Família (USF) do município de João
Pessoa, em virtude de serem campo de estágio dos alunos de graduação em Nutrição da UFPB e por este município não ser local de coleta de dados da pesquisa. Neste momento, registrouse o tempo médio gasto em cada entrevista e identificou-se a necessidade de adaptação de algumas questões que não eram facilmente percebidas pelos entrevistados. As versões reformuladas foram apresentadas em oficina nacional, realizada na cidade de Natal, no estado do Rio Grande do Norte, aos representantes dos estados e municípios participantes da pesquisa.

Esta oficina foi realizada com o objetivo de sensibilizar os participantes quanto à importância do processo avaliativo, bem como para firmar parcerias com estes locais, no intuito de facilitar o processo de coleta de dados e a execução do plano de ação traçado a partir dos resultados obtidos. Além disso, foram definidas as versões finais dos instrumentos.

Foram produzidos instrumentos específicos para cada um dos programas, visando obter informações que subsidiassem o processo avaliativo. Ressalta-se que na construção dos instrumentos objetivou-se também apreender se os conceitos de DHAA e de SAN permeiam a operacionalização dos programas em avaliação. 


\section{Procedimentos de amostragem}

De acordo com o público participante da pesquisa e da técnica para coleta dos dados, foi calculada a amostra, de maneira que esta fosse representativa dos municípios em análise como descrito a seguir.

\section{- Crianças menores de cinco anos} e respectivos responsáveis

Para o município de Cabedelo, a amostra foi do tipo probabilística por conglomerado, com duas etapas de seleção. A primeira compreendeu o sorteio aleatório de quinze das dezenove Unidades de Saúde da Família, que também funcionam como postos de vacinação (os conglomerados). Já nos municípios de Tibau de Sul e Barra de São Miguel, a coleta de dados se deu em todos os postos de vacinação, sendo quatro e três, respectivamente.

Em seguida, com base na previsão do número de crianças a serem imunizadas em cada posto de vacinação dos municípios, de acordo com a primeira etapa da Campanha de Multivacinação, estimou-se o número de responsáveis a serem entrevistados. Como o número de responsáveis não é registrado nas Campanhas de Multivacinação, mas sim o número de crianças imunizadas, considerou-se um responsável para cada duas crianças a serem vacinadas. Foi realizada uma amostragem sistemática, adotando o critério de entrevistar sequencialmente dois responsáveis sim e um não, por ordem de chegada ao posto de vacinação.

Como o número de entrevistas estruturadas inicialmente previsto foi extrapolado, foi necessário equilibrar o total de entrevistas realizadas em cada USF, de acordo com a representatividade percentual no total da amostra de cada município, utilizando um fator de expansão.

No caso de responsáveis com duas ou mais crianças, o instrumento foi direcionado a apenas uma criança. Esta deveria ter o maior grau de proximidade com o entrevistado e/ou ser a mais velha (limite até 59 meses) dentre as presentes. Esta mesma criança teve suas medidas antropométricas aferidas ao final da entrevista.

Foram realizadas 780 entrevistas em Cabedelo, com avaliação nutricional de 750 crianças; 374 entrevistas em Barra de São Miguel, com 371 crianças avaliadas; e 359 entrevistas em Tibau do Sul, com tomada de medidas antropométricas de 356 crianças menores de cinco anos. No total foram entrevistados 1.525 responsáveis, e avaliadas 1.477 crianças.

\section{- Mães de crianças menores de cinco anos participantes dos programas em análise}

As mães de crianças menores de cinco anos do município de Cabedelo que participaram dos encontros de grupos focais foram sorteadas aleatoriamente, a partir de uma lista fornecida pela Coordenação de Alimentação e Nutrição do município. Esta lista continha os nomes de mães de crianças menores de cinco anos acompanhadas por USF. Foram sorteadas dezenove mães, sendo uma de cada área de abrangência das 19 USF do município, no intuito de formar dois grupos. Em virtude da ausência de quatro mães, participaram dos encontros de grupos focais 15 mães.

Em Barra de São Miguel foram sorteadas sete mães por área de abrangência de cada USF existente no município, totalizando 21 mães. Em Tibau do Sul foram sorteadas cinco mães por área de abrangência, totalizando 20 mães. Participaram dos encontros 13 e 16 mães, em Barra de São Miguel e Tibau do Sul, respectivamente.

- Profissionais de saúde - médico(a), enfermeiro(a) e técnico(a) de enfermagem

Para o município de Cabedelo, foi realizado um sorteio aleatório de 09 das 15 USF que serviram de local de coleta de dados na segunda etapa da Campanha de Multivacinação. Nestas USF foram realizadas entrevistas semiestruturadas com 22 profissionais de saúde. Já nos municípios de Tibau do Sul e Barra de São Miguel, foram entrevistados todos os profissionais das USF existentes, totalizando 12 e 09 entrevistas semiestruturadas, respectivamente.

- Profissionais de saúde - nutricionistas inseridos nas equipes de Saúde da Família de Cabedelo

Todos os 10 nutricionistas inseridos nas equipes de Saúde da Família do município de Cabedelo foram convocados a participar tanto das entrevistas estruturadas como do encontro de grupo focal. Participaram das entrevistas estruturadas 09 nutricionistas e 07 do encontro de grupo focal.

- Profissionais de saúde -

Agentes Comunitários de Saúde (ACS)

Os agentes comunitários de saúde do município de Cabedelo que participaram dos encontros de grupos focais foram sorteados aleatoriamente, a partir de uma lista fornecida pela Secretaria de Saúde do município com os nomes de todos os agentes. Foram sorteados 20 ACS no intuito de montar dois grupos, cada um com 10 
participantes. Em virtude da ausência de 05 agentes, participaram dos encontros de grupos focais 15 ACS.

Nos municípios de Barra de São Miguel e Tibau do Sul foram convocados todos os ACS (15 e 20, respectivamente) para os encontros de grupos focais. Participaram destes, 12 e 15 ACS em Tibau e Barra, respectivamente.

\section{- Gestores envolvidos}

\section{na operacionalização dos programas}

Foram realizadas entrevistas semiestruturadas com a Coordenadora da Atenção Básica e a Coordenadora de Alimentação e Nutrição do município de Cabedelo, as quais tinham relação com a gestão do PNVITA neste município. No município de Barra de São Miguel foram realizadas entrevistas semiestruturadas com os Secretários de Saúde e de Ação Social, os Coordenadores do PBF na Saúde e na Ação Social e o digitador-máster do PBF. No município de Tibau do Sul foram realizadas entrevistas semiestruturadas com os Secretários de Saúde e de Ação Social e os Coordenadores do PBF na Saúde e na Ação Social.

\section{Logística montada para a coleta de dados}

Para a realização das entrevistas estruturadas com os responsáveis por crianças menores de cinco anos, foram selecionados e treinados alunos de graduação em Nutrição da UFPB, da Universidade Federal do Rio Grande do Norte e da Universidade Federal de Alagoas. Para a coleta de dados em Cabedelo, foram ainda treinados alunos de graduação em Educação Física os quais realizaram a tomada das medidas antropométricas das crianças. As demais entrevistas e encontros de grupos focais foram realizados pelos pesquisadores.

A supervisão do trabalho de campo foi realizada conjuntamente pela equipe de condução da pesquisa, por representantes das secretarias estaduais e municipais de saúde, e por docentes das universidades parceiras.

\section{Sistematização e análise dos dados coletados}

Os dados coletados foram armazenados utilizando os programas Excel e Word for Windows 2003. A análise estatística dos resultados obtidos foi realizada por meio do programa Epi Info versão 6.04 .

Com relação aos dados obtidos pelas entrevistas estruturadas e semiestruturadas, as ques- tões fechadas geraram tabelas de frequência simples. Já a análise das questões abertas foi realizada nas seguintes etapas:

- identificou-se a ideia central de cada resposta apresentada, baseada no questionamento realizado;

- criaram-se categorias a partir das ideias extraídas das respostas;

- codificaram-se as categorias para análise das respostas.

Com relação aos grupos focais, após a leitura de todo o material produzido (anotações do relator e transcrição, na íntegra, das gravações), os achados foram sistematizados em categorias predefinidas na elaboração dos instrumentos. Em seguida, procedeu-se à análise temática considerando as palavras, o contexto, a frequência, a intensidade dos comentários, a especificidade das respostas e a consistência interna ${ }^{23}$.

Em cada eixo definido procurou-se observar e analisar as fragilidades, as potencialidades e apontar reflexões sobre alternativas para a reorientação e/ou qualificação das ações e serviços de saúde inerentes aos programas em análise e destinados à população-alvo.

Com relação aos dados referentes ao PBF, no recorte com os responsáveis por crianças menores de cinco anos, realizou-se uma análise descritiva comparativa entre as respostas dos participantes e não participantes do programa.

No tocante aos dados antropométricos coletados, foram produzidos os índices estatura/idade, comprimento /idade, peso/idade e peso/altura, os quais foram calculados para cada criança segundo os valores em escore Z. Como população de referência, foram adotadas as Curvas de Crescimento Infantil da Organização Mundial de Saúde ${ }^{24}$. Os dados do levantamento antropométrico foram processados utilizando o programa Anthro 2006.

\section{Resultados e discussão}

\section{Lições aprendidas, reflexões e cuidados metodológicos}

- A utilização do guia metodológico IA-FAO auxiliou na definição das questões que seriam utilizadas nas entrevistas e grupos focais com os diversos atores sociais envolvidos na operacionalização dos programas em análise, bem como serviu de suporte para definição dos eixos de análise que não poderiam deixar de ser considerados tanto no desenho do trabalho de campo como na análise dos dados e informações obtidas; 
- A partir do trabalho de campo percebeu-se que realizar avaliações de programas de alimentação e nutrição (e programas e políticas públicas em geral) em períodos próximos às eleições não é a opção mais oportuna, pois os atores sociais envolvidos se mostram apreensivos com o processo avaliativo e receosos de que os aspectos negativos identificados ganhem destaque e influenciem no processo eleitoral. Assim, considerase estratégico realizar avaliações sempre em início de mudança de governo, diagnosticando a situação atual de funcionamento dos programas, os nós críticos e o impacto favorável desses, para então traçar um plano de ação construído democraticamente (com a participação de gestores, representantes da comunidade e profissionais), com representação intersetorial. É importante destacar que avaliações em início de governo impõem, muitas vezes, que atores da gestão anterior sejam entrevistados, considerando as mudanças de cargos e por vezes o desconhecimento dos novos gestores em relação aos programas que passam a coordenar;

- Identificou-se a inexistência de um processo de monitoramento e avaliação institucionalizado que extrapole estatísticas de produção e incorpore a participação de todos os atores sociais envolvidos nos programas. Assim, é fundamental a estruturação de um protocolo de monitoramento e de avaliações sistemáticas das ações acordadas no plano de ação, que podem contribuir favoravelmente para o aperfeiçoamento dos programas. Além disso, é importante que haja previsão dos governos Federal, Estadual e Municipal de recursos e insumos para a área de monitoramento e avaliação de programas de alimentação e nutrição. A formação e/ou fortalecimento de parcerias com Universidades e entidades governamentais e não governamentais pode ser uma estratégia bastante favorável;

- O primeiro momento da coleta de dados "in loco" nos municípios ocorreu durante a segunda etapa da campanha de multivacinação. A escolha da data foi permeada pela lógica de captar um maior número possível de mães/responsáveis de menores de cinco anos aproveitando a estrutura já montada e bem organizada para as campanhas. No entanto, durante todo o processo de preparação da logística e de treinamento da equipe para aplicação das entrevistas teve-se o cuidado de destacar a importância do seguimento da metodologia programada para alcance da meta e minimização dos riscos de atrapalhar a vacinação. Dessa forma, a logística montada para as entrevistas foi realizada em conjun- to com os responsáveis nos municípios pelo Programa Nacional de Imunização, os quais foram parceiros fundamentais nesse momento. Objetivando tornar o processo o mais tranquilo possível e sem longas esperas por parte das mães (minimizando perdas por desistências), definiu-se que em cada posto de vacinação ficariam entre dois a três entrevistadores a depender da demanda prevista a partir da campanha anterior;

- O trabalho de campo reafirmou que a opção pelo sorteio aleatório por área de abrangência das USF para montagem dos participantes dos grupos focais (mães e agentes comunitários de saúde) foi uma importante opção metodológica, visto que permitiu a apreensão ampliada de percepções diversificadas sobre os programas;

- A cooperação dos municípios foi peça chave para a viabilização do trabalho de campo. Nos três municípios com os programas em análise, os gestores tentaram superar as dificuldades administrativas e contribuíram, sempre que possível, para que tudo fluísse conforme planejado. Destacam-se alguns fatores facilitadores deste processo de cooperação dos municípios: a) $\mathrm{Na}$ seleção dos municípios participantes considerouse "a vontade política" do gestor em aderir ao processo de análise; b) Sensibilização através da oficina nacional realizada para repasse da metodologia IA - FAO com representantes dos municípios; c) O planejamento prévio por parte da equipe de condução do trabalho de campo da logística necessária à coleta de dados. Durante a organização do trabalho de campo foi realizado contato frequente com os nutricionistas e coordenadores dos programas dos municípios para repasse de informações em um processo de corresponsabilização;

- A maioria dos entrevistados percebia os pesquisadores como profissionais que estavam no município com o objetivo de orientá-los quanto aos programas, ou seja, na função de capacitadores, incluindo nesta dinâmica os gestores e profissionais de nível superior. O trabalho de campo foi percebido também como um momento de sensibilização para reorientação das ações, capacitação e troca de informações, tornando-se uma oportunidade dos atores sociais inseridos na análise (mães, agentes comunitários de saúde e os demais profissionais) expressarem suas dúvidas e seus desejos de maior informação para contribuírem com mudanças favoráveis. Dentro do possível, as dúvidas eram esclarecidas pelos pesquisadores, sempre ao final da sessão para minimizar os riscos de enviesar a análise. 


\section{Análise do acompanhamento das condicionalidades de saúde do PBF}

Foram encontrados resultados bastante próximos nos dois municípios estudados com relação ao acompanhamento das condicionalidades de saúde do PBF. As informações demográficas e socioeconômicas evidenciaram a focalização do programa, visto que nas famílias beneficiárias os níveis de escolaridade são mais baixos e o número de pessoas por família é maior do que o de famílias não beneficiárias.

No campo das imunizações, vale destacar que as crianças participantes do PBF tenderam a apresentar maiores coberturas vacinais nas doses tardias e de reforço do que as outras crianças, o que pode estar relacionado com o acompanhamento desta condicionalidade.

Nos dois municípios, somente de 60 a $70 \%$ dos responsáveis por crianças participantes do PBF conheciam as regras gerais do Programa, revelando um desconhecimento de boa parte das famílias. A percepção do impacto da transferência de renda foi positiva, considerando que cerca de $90 \%$ dos entrevistados relataram melhorias nas suas condições de vida.

As famílias referiram relativa facilidade com relação ao cumprimento das condicionalidades de saúde, principalmente em função da interlocução com os Agentes Comunitários de Saúde e o acolhimento pelas USF. Quanto aos problemas referidos, destacou-se a dificuldade de acesso geográfico por parte de algumas famílias participantes do Programa para o acompanhamento pela equipe da unidade de saúde.

Entre os profissionais de saúde, uma das principais dificuldades relatadas foi a ausência de cursos de capacitação a respeito do PBF, mais especificamente em relação ao acompanhamento das condicionalidades de saúde.

\section{Análise do PNVITA}

De modo geral, os resultados evidenciaram que o eixo central do Programa no município estudado é a distribuição de megadoses de vitamina A e que a realização de orientações às famílias, quando existe, é pontual e vinculada a alguns profissionais em particular.

A participação dos responsáveis na operacionalização do programa se mostrou frágil, sendo limitada à presença na USF para administração das cápsulas às suas crianças. Identificou-se, também, falta de conhecimento acerca do Programa por parte dos profissionais das USF, que destacaram a importância de eventos de formação continuada sobre este tema. Quanto à cobertura do programa, observou-se que esta ainda não está adequada a recomendação do Ministério da Saúde do Brasil ${ }^{25}$.

\section{Análise do estado nutricional}

A avaliação nutricional das crianças menores de cinco anos revelou o processo de transição nutricional que vem ocorrendo nos municípios (baixas prevalências de déficit de peso para idade e prevalências mais altas de excesso de peso). Além disso, no município de Tibau do Sul, em que foi analisado o PBF, observaram-se diferenças significativas entre as prevalências dos déficits de altura para idade de beneficiários e não beneficiários mostrando que o referido programa, que tem como critério de inclusão baixa renda per capita familiar, inclui crianças em situação nutricional mais desfavorável.

\section{Plano de ação traçado \\ para a melhoria dos programas analisados}

A partir das fragilidades identificadas no processo avaliativo, foi construído um plano de ação específico para cada um dos programas avaliados, com a participação dos gestores (municipais e estaduais) e profissionais envolvidos na implementação destes em cada município. Estes planos abordaram desde questões operacionais dos programas, como estratégias de registro das informações, de abastecimento de insumos, até questões de capacitação, especialmente voltadas aos profissionais inseridos nas equipes de Saúde da Família, visando à formação de multiplicadores (profissionais - comunidades). As propostas de intervenção contidas nos planos foram direcionadas aos três níveis de gestão.

$\mathrm{Na}$ esfera nacional, recomendou-se a disponibilização de meios para a qualificação de atores da atenção básica para a implementação e controle social de ações e programas de alimentação e nutrição, na ótica do DHAA e SAN, como cursos à distância para profissionais e outros atores sociais que atuam no âmbito do Sistema Único de Saúde (SUS) de todo o país.

$\mathrm{Na}$ esfera estadual, foi apontada a necessidade de promoção de ciclos de capacitações direcionados aos ACS para atuar nas ações de alimentação e nutrição em conjunto com as equipes multiprofissionais da rede básica do SUS, em especial, com as equipes de Saúde da Família, contextualizando-o como um agente promotor da 
alimentação saudável no enfoque da promoção à saúde.

$\mathrm{Na}$ esfera municipal, indicou-se a implementação de estratégias específicas para o aperfeiçoamento da gestão e do acompanhamento dos programas, por meio da formulação de planos de ação locais, construídos democraticamente com a participação de gestores, representantes da comunidade e profissionais de saúde, além de representantes de outros setores.

Considerando as especificidades dos dois programas, em especial no que se refere ao tempo de implementação destes, ao desenho operacional, aos tipos de ações implementadas e aos papéis dos diferentes níveis de governo, os planos de ação contemplaram estratégias específicas para cada programa.

Para o PNVITA as estratégias de ação foram direcionadas prioritariamente à qualificação do processo de registro dos dados, do abastecimento e armazenamento das cápsulas de megadose de vitamina $\mathrm{A}$, além da intensificação das ações educativas voltadas à promoção da alimentação saudável, de forma que esta inclua alimentos-fonte de vitamina A. O objetivo é aperfeiçoar as ações estruturais do Programa (incorporação dos alimentos-fonte de vitamina A na alimentação da população), reduzindo cada vez mais a necessidade da ação emergencial desenvolvida pelo mesmo (distribuição das cápsulas de vitamina A).

Já para o PBF, foram apontadas estratégias voltadas ao compartilhamento das responsabilidades entre os diversos profissionais e gestores do programa, bem como a necessidade de intensificar as ações de educação em saúde, na perspectiva de promover a autonomia dos sujeitos participantes do programa quanto ao autocuidado e corresponsabilização no monitoramento das ações do PBF.

\section{Colaboradores}

AT Carvalho, ER Almeida, EAF Nilson e JA Ubarana participaram na concepção do projeto, na coleta, análise e interpretação dos dados, e na redação do artigo. IM Fernández e M Imink participaram na redação do artigo.

\section{Agradecimentos}

À Organização das Nações Unidas para Agricultura e Alimentação, pelo apoio financeiro. À equipe da Coordenação Geral de Alimentação e Nutrição do Ministério da Saúde, pelo apoio técnico e logístico. Às Secretarias de Saúde e Ação Social dos estados e municípios participantes da pesquisa. Às universidades parceiras, pelo apoio na coleta de dados. 


\section{Referências}

1. Coutinho JG, Gentil PC, Toral N. A desnutrição e obesidade no Brasil: o enfrentamento com base na agenda única da nutrição. Cad Saude Publica 2008; 24(Supl. 2):332-340.

2. Pessanha LDR. A experiência brasileira em politicas públicas para a garantia do direito humano ao alimento. Rio de Janeiro: Escola Nacional de Ciências Estatísticas, Departamento de Ensino e Pesquisa; 2002. (Texto para discussão, no 5)

3. Organização das Nações Unidas para a Agricultura e Alimentação (FAO). Directrices para la Formulación de Planes Nacionales de Acción para la Nutrición. Conferência Internacional sobre Nutrição. Roma: FAO; 1993. [acessado 2010 abr 28].Disponível em: http://www.direitoshumanos.usp.br.

4. Organização das Nações Unidas para a Agricultura e Alimentação (FAO). Declaração de Roma Sobre a Segurança Alimentar Mundial \& Plano de Ação da Cúpula Mundial da Alimentação. Cúpula Mundial de Alimentação. Roma: FAO; 1996.

5. Maluf RS. Segurança alimentar e fome no Brasil 10 anos da Cúpula Mundial de Alimentação. Centro de Referência em Segurança Alimentar e Nutricional [Relatório Técnico]. [acessado 2010 abr 28]. Disponível em: http://www.ufrrj.br/cpda/ceresan/ docs/relatoriotecnico2.pdf

6. Lalonde RJ. Building Capacity to Evaluate Health and Nutrition Programs. J Public Health Management and Practice 2009; 15(Supl. 6):90-99.

7. Paes de Barros R, Carvalho M. Desafio para a politica social brasileira. Brasília: IPEA; 2003. (Textos para Discussão, n. 985).

8. Organização das Nações Unidas para a Agricultura e Alimentação (FAO). Factores determinantes del êxito de los programas de alimentación y nutrición comunitários - Examen y análisis de la experiência. Roma: FAO; 2005.

9. Organização das Nações Unidas para a Agricultura e Alimentação (FAO). Melhoria dos Programas de Nutrição - Um instrumento de Análise para a Ação. Edição Revisada. Roma: FAO; 2006.

10. Brasil. Ministério do Desenvolvimento Social e Combate à Fome. Cidadania: O principal ingrediente do Fome Zero. Brasília: Ministério do Desenvolvimento Social e Combate à Fome; 2005. [acessado 2010 out. 20]. Disponível em: http://www. fomezero.gov.br/publicacoes/publicacoes/arquivos /livreto_fome_zero.pdf.

11. Brasil. Presidência da República. Decreto nº 6.491, de 26 de junho de 2008. Dá nova redação ao art. 19 do Decreto no 5.209, de 17 de setembro de 2004, que regulamenta a Lei no 10.836 , de 9 de janeiro de 2004, que cria o Programa Bolsa Família. Diário Oficial da União 2004; 26 jun.

12. Brasil. Ministério do Desenvolvimento Social e Combate à Fome. Ministério da Saúde. Portaria Interministerial no 2.509 , de 18 de novembro de 2004. [acessado 2010 out 20]. Disponível em: http:/ /dtr2001.saude.gov.br/sas/PORTARIAS/Port2004/ $\mathrm{Gm} / 2509 . \mathrm{htm}$

13. World Health Organization (WHO). Global prevalence of vitamin A deficiency in populations at risk 1995-2005. Geneva: WHO; 2009.
14. Ramalho RA, Flores H, Saunders C. Hipovitaminose A no Brasil: um problema de saúde pública. Rev Panam Salud Publica 2002; 12(2):117-122.

15. Geraldo RRC, Paiva SAR, Pitas AMCS, Godoy I, Campana AO. Distribuição da hipovitaminose A no Brasil nas últimas quatro décadas: ingestão alimentar, sinais clínicos e dados bioquímicos. Rev Nutrição 2003; 16(4):443-460.

16. Brasil. Ministério da Saúde (MS). Portaria no. 729/ GM, de 13 de maio de 2005. [acessado 2010 out. 20]. Disponível em: http://dtr2001.saude.gov.br/sas/ PORTARIAS/Port2005/GM/GM-729.htm.

17. Graebner IT. Níveis plasmáticos de vitamina $A$, ações pedagógicas e segurança alimentar: estudo em escolares rurais do Distrito Federal [tese]. Brasília: Universidade de Brasília; 2007.

18. Pereira JA, Paiva AA, Bergamaschi DP, Rondó PHC, Oliveira GC, Lopes IBM, Illison VK, Gonçalves-Carvalho CMR. Concentrações de retinol e de betacaroteno séricos e perfil nutricional de crianças em Teresina, Piauí, Brasil. Rev bras epidemiol 2008; 11(2): 287-296.

19. Brasil. Ministério da Saúde (MS). Secretaria de Atenção à Saúde. Vitamina A Mais: Programa Nacional de Suplementação de Vitamina A - condutas gerais. Brasília: MS; 2004.

20. Brasil. Ministério da Saúde (MS). Pesquisa Nacional de Demografia e Saúde da Criança e da MulherPNDS 2006: dimensões do processo reprodutivo e da saúde da criança. Brasília: MS, Centro Brasileiro de Análise e Planejamento; 2009.

21. Minayo MC, Neto O. Triangulación de métodos en la evaluación de programas y servicios de salud. In: Bronfman J, Castro R, organizadors. Salud, cambio social y política - Perspectivas desde América Latina. México: Insp, Edamex; 1999. p. 65-80.

22. Boni V, Quaresma SJ. Aprendendo a entrevistar: como fazer entrevistas em ciências sociais. Rev Eletrôn dos Pós-Graduandos Sociol Polit UFSC 2005; 2(1): 68-80.

23. Minayo MCS. O Desafio do conhecimento: pesquisa qualitativa em saúde. São Paulo: Hucitec, Abrasco; 2000.

24. World Health Organization, Food and Agriculture Organization of the United Nations. Guidelines on food fortication with micronutrients. Geneva: WHO; 2006. [acessado 2010 out. 25]. Disponível em: http:/ /www.who.int/nutrition/publications/micronutrients /guide_food_fortification_micronutrients.pdf.

25. Almeida ER, Carvalho AT, Nilson EAF, Coutinho JG, Ubarana JA. Avaliação participativa do Programa Nacional de Suplementação de Vitamina A em um município da Região Nordeste do Brasil. Cad Saude Publica 2010; 26(5):949-960.

Artigo apresentado em 14/04/2011

Aprovado em 10/08/2011

Versão final aprovada em 30/11/2011 